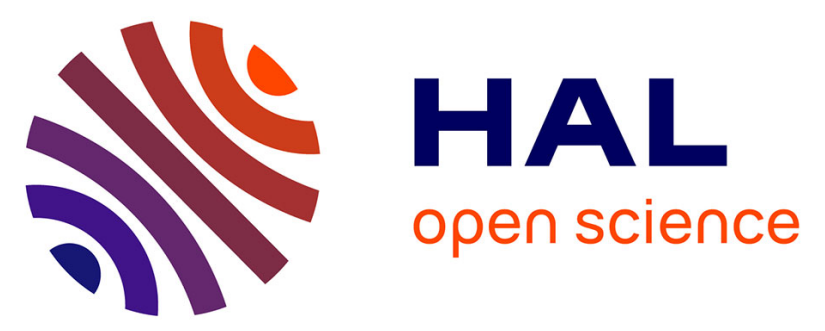

\title{
Practical indicators for assessing the magnitudes of wall radiative flux and of coupling effects between radiation and other heat transfer modes on the temperature law-of-the wall in turbulent gaseous boundary layers
}

\author{
Y. F. Zhang, Ronan Vicquelin, O. Gicquel, J. Taine
}

\section{To cite this version:}

Y. F. Zhang, Ronan Vicquelin, O. Gicquel, J. Taine. Practical indicators for assessing the magnitudes of wall radiative flux and of coupling effects between radiation and other heat transfer modes on the temperature law-of-the wall in turbulent gaseous boundary layers. International Journal of Heat and Mass Transfer, 2018, 120, pp.76 - 85. 10.1016/j.ijheatmasstransfer.2017.12.026 . hal-01744490

\author{
HAL Id: hal-01744490 \\ https://hal.science/hal-01744490
}

Submitted on 27 Mar 2018

HAL is a multi-disciplinary open access archive for the deposit and dissemination of scientific research documents, whether they are published or not. The documents may come from teaching and research institutions in France or abroad, or from public or private research centers.
L'archive ouverte pluridisciplinaire HAL, est destinée au dépôt et à la diffusion de documents scientifiques de niveau recherche, publiés ou non, émanant des établissements d'enseignement et de recherche français ou étrangers, des laboratoires publics ou privés. 


\title{
Practical indicators for assessing the magnitudes of wall radiative flux and of coupling effects between radiation and other heat transfer modes on the temperature law-of-the wall in turbulent gaseous boundary layers
}

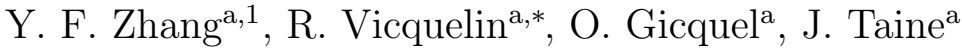 \\ ${ }^{a}$ Laboratoire EM2C, CNRS, CentraleSupélec, Université Paris-Saclay, 3 rue Joliot \\ Curie, 91192 Gif-sur-Yvette cedex, France.
}

\begin{abstract}
Practical indicators are derived to compare radiative and conductive fluxes and the coupling effects of conduction, convection and radiation in turbulent gaseous boundary layers. A first criterion controlling the weight of wall radiative flux compared to wall conductive flux is introduced to assess the necessity of performing radiation simulation under given flow conditions. A second criterion based on the variation of the non-dimensional temperature due to radiation (scaled in wall units) is also developed to predict whether a wall model accounting for radiation is required when coupling effects become significant. These criteria are built from turbulent channel gaseous flow field simulations for many typical conditions, based on a $k$ - $\epsilon$ model, a given turbulent Prandtl number expression and a spectal ck model for radiation. They have been validated from the comparison with corresponding fully-coupled results where turbulent fields are solved numerically with direct numerical simulations and where the radiative energy transfer is solved with a Monte Carlo approach.

The obtained criterion results are then presented and thoroughly analyzed on a large set of conditions, which would not have been feasible with direct numerical simulations or large-eddy simulations. The influence of system size,
\end{abstract}

\footnotetext{
*Corresponding author. Tel.: +33 175316090

Email address: ronan.vicquelin@centralesupelec.fr (R. Vicquelin)

${ }^{1}$ Current address: AECC Commercial Aircraft Engine CO., LTD., Shanghai, 200241, P.R. China
} 
Reynolds number, wall emissivity and pressure is synthesized in $2 \mathrm{D}$ contour plots of interest for engineers and researchers to assess the magnitude of radiation effects in wall bounded flows.

Keywords: Gas radiation, turbulent boundary layers, wall models

\section{Introduction}

In industrial systems such as those involving combustion, the wall heat flux due to hot burnt gases is a key quantity which is even more critical as the considered temperature and pressure increase. In such conditions, both conductive and radiative wall fluxes, and possibly the coupling effects between the different heat transfer mechanisms must be accounted for. An accurate determination of the wall heat flux field is therefore a priori based on a simulation of coupled heat conduction, convection and thermal radiation. Such an approach requires to solve the radiative transfer equation along with accurate radiative properties of burnt gases. This simulation that would capture radiation effects and their coupled effects with other heat transfer mechanisms significantly increases the computational time. From a practical point of view, it is interesting to estimate beforehand these effects in order to determine whether such coupled simulations are necessary. This is the objective of the present study which focuses on the reciprocal couplings between conduction, convection and radiation in turbulent gaseous boundary layers.

The channel flow configuration is a canonical case of great interest to study turbulent boundary layers in internal flows. The effects of radiation have then been investigated in such a configuration in several studies with different levels of description of the turbulent flow field and radiation in the participating medium. Soufiani et al. [1] have studied such effects with RANS simulations combined with radiation computed from the statistical narrow-band model (SNB). In addition to the resulting supplemental wall radiative flux, it has been observed that radiation also affects the wall conductive flux because of the modified temperature profile. With the increase of computational resources, more accurate methods have been used to describe turbulent flows. Amaya et al. [2] have reported results from a direct numerical simulation of turbulence (DNS) coupled to a discrete ordinate method to solve radiative energy transfer with a global spectral model [3]. For the investigated case, the effect of radiation in the thermal boundary layer is weak. Gupta et al. [4] have carried out large-eddy simulation (LES) of a 
turbulent channel flow and have solved the radiative transfer equation with a spherical harmonics (P1) method and a grey gas model. They have showed that depending on the medium optical thickness, the temperature profile in the turbulent channel flow can be modified by radiation. Supersonic channel flows with radiation have been investigated in Ref.[5] where radiative properties have been described with a fictitious grey gas and a CK model [6]. Mixed convection in a horizontal channel has been studied by Sakurai et al. [7] by considering radiation under the optically thin limit.

In order to analyse simulation results as accurate as possible, Zhang et al. [8] have carried out direct numerical simulations of turbulent channel flows coupled with an accurate gas radiation model, based on a reciprocal Monte-Carlo method [9] and a ck model of radiative properties [10, 11] whose coefficients have been determined from line-by-line calculations. This extensive study has covered several parameter variation ranges: wall temperature, pressure, wall emissivity, Reynolds number. Under some conditions, the wall radiative flux, which depends on the turbulent and mean temperature fields, is not negligible. Moreover, thermal radiation can also significantly modify the temperature turbulent field, its mean profile and then the wall conductive flux $[8,12]$. The analysis of these reciprocal coupling effects have highlighted the role of radiative transfer. Gas-gas radiative interactions tend to homogenize the temperature field. The resulting steepening of the mean temperature profile close to the wall increases the wall conductive flux. On the other hand, gas-wall radiative interactions have the opposite effect. Such results have a strong impact on wall modeling in RANS and large-eddy simulations since classical wall laws are not valid anymore. A specific wall model has successfully been derived and validated [13] to deal with these radiation effects. While considered for LES, the same model can be implemented in RANS computations.

In summary, radiation effects in turbulent boundary layers result in an additional wall radiative flux and a possible modification of the universal temperature profile known as the law-of-the-wall. Accounting for the latter effect requires a specific wall model. For both effects, it is useful to build costless indicators to know whether it is necessary to account for radiation and to use a specific wall model. This is the objective of the present study which considers two indicators computed from one-dimensional mean temperature profiles. These profiles are determined from 1D RANS computations without being coupled to the resolution of the radiative transfer equation. The first indicator compares the wall radiative flux to the wall conductive flux 
in order to determine whether a radiation simulation should be carried out. The second indicator assesses the necessity of a wall-model to account for the modification of the temperature law-of-the-wall due to radiation effects. Both indicators are defined in Sec. 2. Their determination from simulations without radiation is assessed on the database of direct numerical simulations of channel flows carried out by Zhang et al. [8] carried out with and without radiation. The practical computation of the indicators is then based on 1D RANS equations which are detailed in Sec. 3. Finally, the computed indicators are shown and analyzed for a wide variations of parameters (channel width, Reynolds number, wall emissivity, pressure) that are assembled in two-dimensional maps allowing to identify the magnitude and nature of radiation effects in various conditions.

\section{Indicators for radiation effects in turbulent boundary layers}

Since computing the radiation field is generally very expensive in computational ressources, it is interesting to predict the importance of radiation before performing an accurate simulation. In this section, several criteria are proposed in order to determine whether it is necessary to simulate the radiation field and further, to predict if the wall model proposed in Ref. [13] is required under the studied flow conditions.

\subsection{Indicator quantifying the wall radiative flux}

The averaged wall heat flux is the sum of the averaged wall conductive heat flux $\overline{q_{w}^{c d}}$ and radiative flux $\overline{q_{w}^{R}}$. The latter flux can be written as

$$
\overline{q_{w}^{R}}=\overline{q_{w g}^{R}}+\overline{q_{w w}^{R}},
$$

where $\overline{q_{w w}^{R}}$ is the averaged flux exchanged between the walls through the whole gaseous medium, which does not participate to the fluid energy balance and

is zero if the walls are at the same temperature; $\overline{q_{w g}^{R}}$ is the averaged wall flux due to the radiative exchange between the walls and the gas. It is worth noticing that $\overline{q_{w}^{c d}}$ is a priori strongly coupled to radiation and reciprocally $\overline{q_{w}^{R}}$, $\overline{q_{w g}^{R}}$ and $\overline{q_{w w}^{R}}$ are strongly coupled to conduction and convection. By omitting the passive wall-wall radiation contribution, the importance of averaged wall radiative flux compared to the conductive one is evaluated with the following criterion

$$
\mathcal{I}=\frac{\overline{q_{w g}^{R}}}{\overline{q_{w}^{c d}}} .
$$




\begin{tabular}{c|ccccc}
\hline & $\operatorname{Re}_{D_{h}}$ & $T_{w, c}[\mathrm{~K}]$ & $T_{w, h}[\mathrm{~K}]$ & $\mathrm{p}[\mathrm{atm}]$ & $\epsilon_{w}$ \\
\hline $\mathrm{C} 1 \mathrm{R} 1$ & 23400 & 950 & 1150 & 40.0 & 0.8 \\
\hline $\mathrm{C} 2 \mathrm{R} 1$ & 23400 & 950 & 1150 & 1.0 & 0.8 \\
\hline $\mathrm{C} 2 \mathrm{R} 2$ & 23400 & 950 & 1150 & 1.0 & 0.3 \\
\hline $\mathrm{C} 2 \mathrm{R} 3$ & 23400 & 950 & 1150 & 1.0 & 0.1 \\
\hline $\mathrm{C} 3 \mathrm{R} 1$ & 23400 & 950 & 2050 & 40.0 & 0.8 \\
\hline $\mathrm{C} 4 \mathrm{R} 1$ & 47000 & 950 & 1150 & 40.0 & 0.8 \\
\hline
\end{tabular}

Table 1: Channel flow parameters from DNS simulations in Ref. [8].

This comparative quantity of wall heat transfer mechanisms is similar to the conduction-to-radiation parameter introduced in numerous studies [14]. Here, the exact wall conductive flux is considered along with the wall radiative flux only emitted by the gas. The exact averaged wall quantities $\overline{q_{w g}^{R}}$ and $\overline{q_{w}^{c d}}$ that define this indicator require the knowledge of all coupled fields. Radiation modifies the instantaneous and mean temperature fields that determine both $\overline{q_{w}^{c d}}$. Reciprocally, the turbulent temperature field modifies the mean and instantaneous radiation fields and then $\overline{q_{w g}^{R}}$. These phenomena are known as turbulence-radiation interaction [15]. Without any models, the determination of the indicator $\mathcal{I}$ requires the knowledge of the coupled time-varying and 3D temperature field.

However, since the objective of the criterion is to predict the importance of radiative flux before carrying out a real coupled radiation/flow field simulation, the quantities $\overline{q_{w g}^{R}}$ and $\overline{q_{w}^{c d}}$ are not available. Another criterion can therefore be defined from simple approximations: the averaged uncoupled wall-gas radiative flux $\left.\overline{q_{w g}^{R}}\right|_{n c}$ and wall conductive flux $\left.\overline{q_{w}^{c d}}\right|_{n c}$ are determined from the mean temperature field $\bar{T}$ obtained without accounting for radiation. This approximated criterion is then written as

$$
\widetilde{\mathcal{I}}=\frac{\left.\overline{q_{w g}^{R}}\right|_{n c}}{\left.\overline{q_{w}^{c d}}\right|_{n c}} .
$$

The two indicators $\mathcal{I}$ and $\widetilde{\mathcal{I}}$ are compared for the channel flow cases investigated in Ref. [8] with coupled direct numerical simulations. The considered channel flow configurations are given in Tab. 1. The Reynolds number $\operatorname{Re}_{D_{h}}$ is defined from the channel bulk velocity and hydraulic diameter. The mix- 
ture composition is uniform with the $\mathrm{CO}_{2}, \mathrm{H}_{2} \mathrm{O}$ and $\mathrm{N}_{2}$ molar fractions of $0.116,0.155$ and 0.729 , respectively. The considered mixture flows between two plane walls: one at a cold temperature $T_{w, c}$ and the other at a hot temperature $T_{w, h}$, which enables to study two different boundary layers at the same time in a given simulation. The wall emissivity is denoted by $\epsilon_{w}$. The case $\mathrm{C}_{n} \mathrm{R}_{m}$ designates a specific flow configuration $\mathrm{C}_{n}$ (wall temperature, pressure, Reynolds number) associated to a specific setup $R_{m}$ for the wall emissivity. In Ref. [8], pressure effects have been studied by comparing cases C1R1 (40 atm) and C2R1 (1 atm). Cases C2R1, C2R2, C2R3 are distinguished by the considered wall emissivity. Finally, temperature and Reynolds effects have been investigated by comparing the case C1R1 with cases C3R1 and C4R1, respectively. The DNS data whose analysis has been summarized in the introduction provide reference results for different conditions (wall temperature, pressure, wall emissivity, Reynolds number) to assess the relevancy of the uncoupled indicator $\widetilde{\mathcal{I}}$. Results are presented in Fig. 1 where each symbol corresponds to one side (cold or hot wall) of the computed channel flow in a different case. The indicator $\mathcal{I}$ is computed from the coupled DNS results while the indicator $\widetilde{\mathcal{I}}$ is computed from DNS results without radiation. The dashed line added in the figure would correspond to both indicators being identical. Figure 1 shows that, for all cases, values of the two criteria are highly correlated. The indicator $\widetilde{\mathcal{I}}$ can then be used to roughly predict the contribution of the averaged wall radiative flux to the total wall heat flux instead of $\mathcal{I}$.

\subsection{Indicator quantifying the validity of the temperature law-of-the-wall}

Under some conditions, radiation strongly modifies the temperature lawof-the-wall in a turbulent boundary layer. An accurate determination of the relative variation of the scaled mean temperature profile, $\frac{\bar{T}^{+}-\left.\bar{T}^{+}\right|_{n c}}{\left.\bar{T}^{+}\right|_{n c}}$, within the boundary layer due to the coupling with radiation, has first been accurately achieved in typical cases by a turbulence DNS coupled to a radiation Monte Carlo model in Ref. [8]. In the previous ratio, the fields $\bar{T}^{+}$and $\left.\bar{T}^{+}\right|_{n c}$ are issued from coupled and non coupled calculations, respectively.

As such a detailed approach is not suitable in common cases, a practical indicator, based on an approximated expression of $\frac{\bar{T}^{+}-\left.\bar{T}^{+}\right|_{n c}}{\left.\bar{T}^{+}\right|_{n c}}$, will be introduced in this Section to assess the necessity of a specific wall model [13] to account for a possible coupling with radiation. It is based on an approximated 


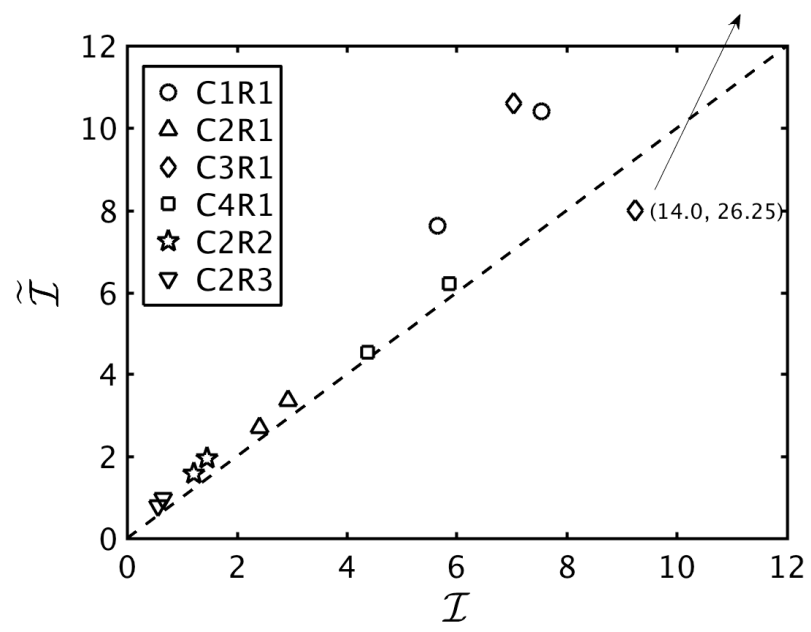

Figure 1: Comparison of indicators quantifying the wall radiative flux: the $\mathcal{I}$ indicator given by Eq. (2) based on coupled results and the approximating indicator $\widetilde{\mathcal{I}}$ indicator given by Eq. (3). Symbols correspond to results (for both cold and hot sides) of coupled direct numerical simulations in different channel flow conditions denoted by C1R1, C2R1, C3R1, C4R1, C2R2 and C2R3 (see Ref. [8]). The dashed line denotes the identity line.

model of the boundary layer, more precisely of its inner region, introduced in Sec. 2.2.1.

\subsubsection{Coupled boundary layer model}

In the inner region of the boundary layer, such that the wall distance $y$ is small compared to the channel half-width $\delta(y \leq 0.1 \delta)$, the mean balance equation of energy is determined by the following expression $[16,17]$ by omitting viscous heating and unsteadiness:

$$
\frac{\mathrm{d}}{\mathrm{d} y}\left(\overline{\lambda \frac{\mathrm{d} T}{\mathrm{~d} y}}+\lambda_{t} \frac{\mathrm{d} \bar{T}}{\mathrm{~d} y}\right)+\overline{P^{R}}=0
$$

where all quantities are issued from coupled fields. $P^{R}=-\frac{\partial q_{j}^{R}}{\partial x_{j}}$ is the radiative power per unit volume, $\lambda$ and $\lambda_{t}$ are the molecular and turbulent thermal conductivity, respectively. After neglecting the effects of properties fluctuations $[18,19]$ and introducing the molecular and turbulent Prandtl number, $\operatorname{Pr}$ and $\operatorname{Pr}_{t}$ respectively, the energy equation is written as

$$
\frac{\mathrm{d}}{\mathrm{d} y}\left[\overline{c_{p}}\left(\frac{\bar{\mu}}{\operatorname{Pr}}+\frac{\mu_{t}}{\operatorname{Pr}_{t}}\right) \frac{\mathrm{d} \bar{T}}{\mathrm{~d} y}\right]+\overline{P^{R}}=0
$$


where $\mu$ and $c_{p}$ are the dynamic viscosity and the thermal capacity at constant pressure. The fluid mass density is denoted by $\rho . \mu_{t}$ is the turbulent viscosity.

Even without radiation, the variations of mean properties $\left(\overline{c_{p}}, \bar{\rho}, \bar{\mu}\right)$ affect the normalized mean temperature profile $\bar{T}^{+}$when using a classical wall scaling based on wall quantities ${\overline{c_{p}}}_{w}, \bar{\rho}_{w}$ and $\bar{\mu}_{w}$. In order to correct these effects, a semi-local scaling $[12,19,20]$ is here retained. Non-dimensional turbulent viscosity $\mu_{t}^{+}=\mu_{t} / \mu$, non-dimensional distance $y^{+}$, based on the wall distance $y$, and the non-dimensional temperature $\bar{T}^{+}$are then defined based on the local value of the fluid properties, i.e.

$$
y^{+}=\frac{\bar{\rho} y u_{\tau}}{\bar{\mu}}, \bar{T}^{+}=\frac{\left|\bar{T}-T_{w}\right|}{T_{\tau}},
$$

where $T_{\tau}$ is the semi-local friction temperature which is defined by

$$
T_{\tau}=\frac{\left|\overline{q_{w}^{c d}}\right|}{\bar{\rho} \overline{c_{p}} u_{\tau}}, \quad u_{\tau}=\left(\frac{\overline{\tau_{w}}}{\bar{\rho}}\right)^{1 / 2}
$$

$\tau_{w}$ is the wall shear stress and $u_{\tau}$ the semi-local friction velocity.

After integration and normalization, Eq. (5) then becomes

$$
\left(\frac{1}{\operatorname{Pr}}+\frac{\mu_{t}^{+}}{\operatorname{Pr}_{t}}\right) \frac{\mathrm{d} \bar{T}^{+}}{\mathrm{d} y^{+}}=1-\frac{\delta \overline{q^{R}}}{\overline{q_{w}^{c d}}},
$$

where $\delta \overline{q^{R}}$ is the variation of the averaged coupled radiative flux $\overline{q^{R}}(y)$ between $y^{+}$and a wall $\left(y^{+}=0\right)$ given by

$$
\begin{aligned}
\delta \overline{q^{R}}\left(y^{+}\right) & =-\int_{0}^{y} \overline{P^{R}}\left(y^{\prime}\right) d y^{\prime} \\
& =\overline{q^{R}}\left(y^{+}\right)-\overline{q_{w}^{R}}
\end{aligned}
$$

Note that, when the effects of coupling with radiation are neglected, Eq. (8) turns into

$$
\left(\frac{1}{\operatorname{Pr}}+\frac{\left.\mu_{t}^{+}\right|_{n c}}{\left.\operatorname{Pr}_{\mathrm{t}}\right|_{n c}}\right) \frac{\left.\mathrm{d} \bar{T}^{+}\right|_{n c}}{\mathrm{~d} y^{+}}=1,
$$

which yields the classical temperature law-of-the-wall in the inner layer, involving uncoupled quantities $\left(\left.\mu_{t}^{+}\right|_{n c},\left.\operatorname{Pr}_{\mathrm{t}}\right|_{n c}\right.$ and $\left.\left.\bar{T}^{+}\right|_{n c}\right)$. 
If the change of $\mu_{t}^{+} / \operatorname{Pr}_{t}$ due to radiation is neglected (although it can be significant [12]), an approximation of the coupled temperature field $\left.\bar{T}^{+}\right|_{\text {app }}$ is determined by solving

$$
\left(\frac{1}{\operatorname{Pr}}+\frac{\left.\mu_{t}^{+}\right|_{n c}}{\left.\operatorname{Pr}_{t}\right|_{n c}}\right) \frac{\left.\mathrm{d} \bar{T}^{+}\right|_{a p p}}{\mathrm{~d} y^{+}}=1-\frac{\delta \overline{q^{R}}\left(y^{+}\right)}{\overline{q_{w}^{c d}}},
$$

The difference of wall-scaled temperatures between models without and with radiation can then be approximated by $\left.\bar{T}^{+}\right|_{n c}-\left.\bar{T}^{+}\right|_{\text {app }}$ given by Eq. (8) and Eq. (12), as

$$
\left(\frac{1}{\operatorname{Pr}}+\frac{\left.\mu_{t}^{+}\right|_{n c}}{\left.\operatorname{Pr}_{\mathrm{t}}\right|_{n c}}\right) \frac{\mathrm{d}}{\mathrm{d} y^{+}}\left(\left.\bar{T}^{+}\right|_{n c}-\left.\bar{T}^{+}\right|_{a p p}\right) \approx \frac{\delta \overline{q^{R}}\left(y^{+}\right)}{\overline{q_{w}^{c d}}}
$$

The relative change of the wall-scaled temperature profile due to radiation is then approximated by integration of Eq. (13), i.e., as the wall temperature is fixed,

$$
\frac{\left.\bar{T}^{+}\right|_{n c}-\left.\bar{T}^{+}\right|_{a p p}}{\left.\bar{T}^{+}\right|_{n c}}\left(y^{+}\right) \approx \frac{1}{\left.\bar{T}^{+}\right|_{n c}\left(y^{+}\right)} \int_{0}^{y^{+}} \frac{1}{\frac{1}{\operatorname{Pr}}+\frac{\left.\mu_{t}^{+}\right|_{n c}}{\left.\operatorname{Pr}_{t}\right|_{n c}}} \frac{\delta \overline{q^{R}}\left(y^{\prime+}\right)}{\overline{q_{w}^{c d}}} \mathrm{~d} y^{\prime+} .
$$

The position $y=0.1 \delta$ (limit of the inner layer in an equilibrium turbulent boundary layer without radiation) is here chosen to quantify the importance of this change and the second criterion $\mathcal{J}$ is defined as

$$
\mathcal{J}=\frac{1}{\left.\bar{T}^{+}\right|_{n c}\left(0.1 \delta^{+}\right)} \int_{0}^{0.1 \delta^{+}} \frac{1}{\frac{1}{\operatorname{Pr}}+\frac{\left.\mu_{t}^{+}\right|_{n c}}{\left.\operatorname{Pr}_{t}\right|_{n c}}} \frac{\delta \overline{q^{R}}\left(y^{++}\right)}{\overline{q_{w}^{c d}}} \mathrm{~d} y^{\prime+} .
$$

For the channel flow cases of Ref. [8], the approximated values given by the previous indicator $\mathcal{J}$ in Eq. (15) are compared in Fig. 2 with the exact relative difference $\left.\frac{\left.\bar{T}^{+}\right|_{n c}-\bar{T}+}{\left.\bar{T}^{+}\right|_{n c}}\right|_{y=0.1 \delta}$, issued from DNS results of Ref. [8] with or without coupling to the Monte Carlo approach for radiation. The two types of results are close.

\subsubsection{Practical indicator for the coupling effects on the temperature law-of- the-wall}

As results accounting for coupling with radiation (i.e. $\delta \overline{q^{R}}\left(y^{+}\right)$and $\overline{q_{w}^{c d}}$ ) are generally not available, a practical crude indicator based only on fields 


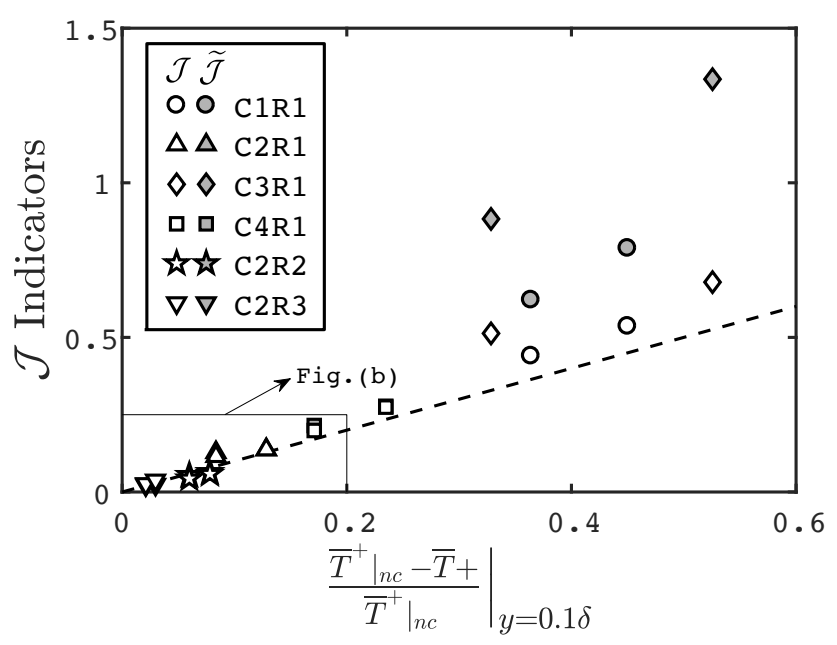

(a)

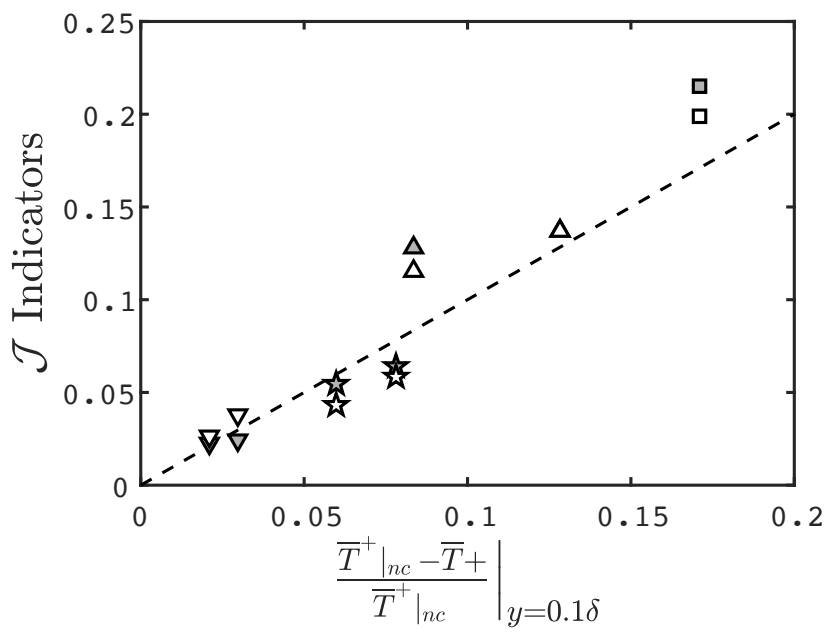

(b)

Figure 2: Comparison of indicators determining the validity of the classical temperature law-of-the wall which is quantified by the relative difference $\left.\frac{\left.\bar{T}^{+}\right|_{n c}-\bar{T}+}{\left.\bar{T}^{+}\right|_{n c}}\right|_{y=0.1 \delta}$. Symbols correspond to results (for both cold and hot sides) of coupled direct numerical simulations in different channel flow conditions denoted by C1R1, C2R1, C3R1, C4R1, C2R2 and C2R3 (see Ref. [8]). White symbols: results with the approximating $\mathcal{J}$ indicator given by Eq. (15) partially based on coupled results. Gray symbols: results with the approximating $\widetilde{\mathcal{J}}$ indicator given by Eq. (16) based on uncoupled results. The dashed line denotes the identity line. 
which are not coupled with radiation is therefore introduced, as done similarly in Sec. 2.1:

$$
\tilde{\mathcal{J}}=\frac{1}{\left.\bar{T}^{+}\right|_{n c}\left(0.1 \delta^{+}\right)} \int_{0}^{0.1 \delta^{+}} \frac{1}{\frac{1}{\operatorname{Pr}}+\frac{\left.\mu_{t}^{+}\right|_{n c}}{\left.\operatorname{Pr}_{t}\right|_{n c}}} \frac{\left.\delta \overline{q^{R}}\right|_{n c}\left(y^{\prime+}\right)}{\overline{\left.q_{w}^{c d}\right|_{n c}}} \mathrm{~d} y^{\prime+} .
$$

where the radiative flux variation $\left.\delta \overline{q^{R}}\right|_{n c}\left(y^{+}\right)$between the position $y^{+}$and the wall is calculated from the mean temperature field of the model without radiation.

The results associated with this practical indicator are also plotted in Fig. 2. Although the agreement between the results associated with $\tilde{\mathcal{J}}$ and with $\left.\frac{\left.\bar{T}^{+}\right|_{n c}-\bar{T}^{+}}{\left.\bar{T}^{+}\right|_{n c}}\right|_{y=0.1 \delta}$ determined by the DNS-Monte Carlo approach is poorer than in the case of $\mathcal{J}$, the trends are correct: a higher indicator value implies a larger change of $\bar{T}^{+}$due to the coupling with radiation. Hence, the criterion $\widetilde{\mathcal{J}}$ based only on an uncoupled model, can be used for determining if a complete coupled approach has to be undertaken.

\section{Numerical model for uncoupled flow field}

A parametric study in channel flows, based on the two previous practical criteria, is carried out in Sec. 4 to identify conditions where radiative energy transfer or associated coupling effects cannot be neglected. The evaluation of both criteria is done from a $1 \mathrm{D}$ mean temperature profile whose determination is detailed in this section. Radiation is not accounted for, yielding a low computational cost of the indicators. The computation of the temperature profile relies on an empirical expression of the turbulent Prandtl number and a turbulent viscosity $\mu_{t}$ determined from the mean velocity field.

\subsection{Equations for the mean velocity field}

The mean velocity field is simulated by the $k-\epsilon$ model from Ref. [21], where $k$ is the turbulent kinetic energy and $\epsilon$ is the turbulent kinetic energy dissipation. The corresponding following equations are numerically solved from a second-order finite volume scheme [22] and a Thomas algorithm applied to the linearized system, which is repeated until convergence, i.e.

$$
\frac{\mathrm{d}}{\mathrm{d} y}\left[\left(\bar{\mu}+\mu_{t}\right) \frac{\mathrm{d} \bar{u}}{\mathrm{~d} y}\right]+S_{u}=0
$$




$$
\begin{gathered}
\frac{\mathrm{d}}{\mathrm{d} y}\left[\left(\bar{\mu}+\frac{\mu_{t}}{\sigma_{k}}\right) \frac{\mathrm{d} k}{\mathrm{~d} y}\right]+\mu_{t}\left(\frac{\mathrm{d} \bar{u}}{\mathrm{~d} y}\right)^{2}-\bar{\rho} \epsilon=0, \\
\frac{\mathrm{d}}{\mathrm{d} y}\left[\left(\bar{\mu}+\frac{\mu_{t}}{\sigma_{\epsilon}}\right) \frac{\mathrm{d} \epsilon}{\mathrm{d} y}\right]+C_{\epsilon 1} \frac{\epsilon}{k} \mu_{t}\left(\frac{\mathrm{d} \bar{u}}{\mathrm{~d} y}\right)^{2}-C_{\epsilon 2} f_{\epsilon} \frac{\bar{\rho} \epsilon^{2}}{k}=0,
\end{gathered}
$$

where $S_{u}$ is the momentum source term, which acts as a pressure gradient term, and drives the flow to a target Reynolds number $\operatorname{Re}_{D_{h}}^{t}$ based on the flow bulk velocity and the hydraulic diameter $D_{h}$. $k$ is the turbulent kinetic energy and $\epsilon$ is the turbulent kinetic energy dissipation. The turbulent viscosity $\mu_{t}$ is computed as

$$
\mu_{t}=C_{\mu} f_{\mu} \frac{\bar{\rho} k^{2}}{\epsilon}
$$

with

$$
\begin{gathered}
f_{\mu}=\left[1-\exp \left(-\frac{y *}{14}\right)\right]^{2}\left\{1+\frac{5}{\operatorname{Re}_{t}^{3 / 4}} \exp \left[-\left(\frac{\mathrm{Re}_{t}}{200}\right)^{2}\right]\right\}, \\
y^{*}=\frac{\bar{\rho} u_{\epsilon} y}{\bar{\mu}} \quad \text { with } u_{\epsilon}=(\bar{\nu} \epsilon)^{1 / 4} \\
\operatorname{Re}_{t}=\frac{\bar{\rho} k^{2}}{\bar{\mu} \epsilon} .
\end{gathered}
$$

The model function $f_{\epsilon}$ is given by

$$
f_{\epsilon}=\left[1-\exp \left(-\frac{y *}{3.1}\right)\right]^{2}\left\{1-0.3 \exp \left[-\left(\frac{\mathrm{Re}_{t}}{6.5}\right)^{2}\right]\right\}
$$

while the model constants are as follow: $C_{\mu}=0.09, \sigma_{k}=1.4, \sigma_{\epsilon}=1.4, C_{\epsilon 1}=$ $1.5, C_{\epsilon 2}=1.9$. The wall boundary conditions for Eq. (17), (18) and (19) are: $\bar{u}_{w}=0.0, k_{w}=0.0, \epsilon_{w}=2 \nu_{w}\left(\partial k^{1 / 2} / \partial y\right)_{w}^{2}$.

\subsection{Equations for the mean temperature field}

The temperature field is then calculated by solving the energy equation:

$$
\frac{\mathrm{d}}{\mathrm{d} y}\left[\overline{c_{p}}\left(\frac{\bar{\mu}}{\operatorname{Pr}}+\frac{\mu_{t}}{\operatorname{Pr}_{\mathrm{t}}}\right) \frac{\mathrm{d} \bar{T}}{\mathrm{~d} y}\right]+S_{T}=0
$$

where the turbulent Prandtl number $\operatorname{Pr}_{\mathrm{t}}$ is modeled as [23]

$$
\operatorname{Pr}_{\mathrm{t}}=\frac{1}{0.5882+0.228\left(\mu_{t} / \bar{\mu}\right)-0.0441\left(\mu_{t} / \mu\right)^{2}\left(1-\exp \left(\frac{-5.165}{\mu_{t} / \bar{\mu}}\right)\right)}
$$




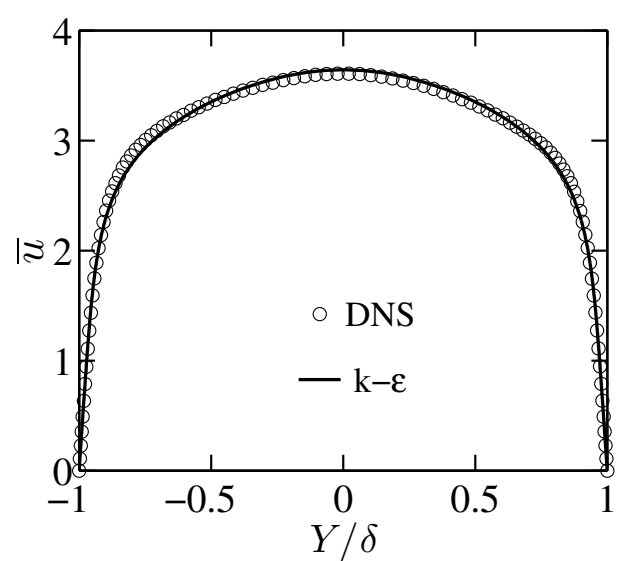

(a)

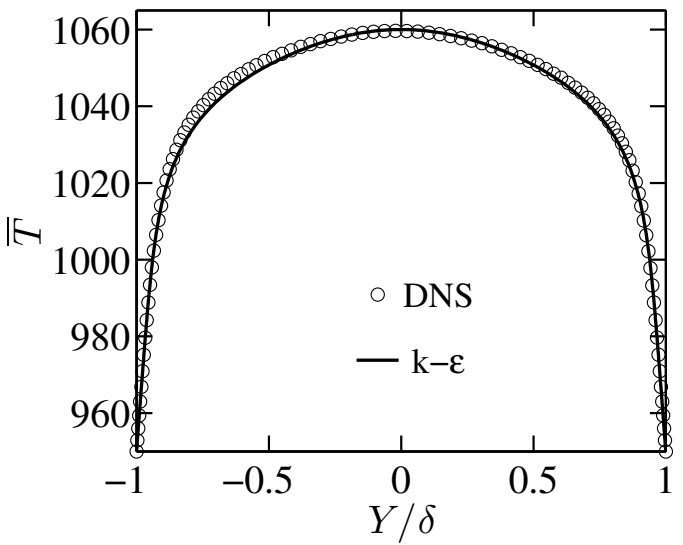

(b)

Figure 3: RANS profiles (plain line) determined from model equations in Secs. 3.1-3.2 of velocity (a) and temperature (b) compared to the DNS results (symbols) by Kim and Moin [24].

and the prescribed wall temperatures are used as boundary conditions. For cases with identical wall temperatures, a source term $S_{T}$ is added in Eq. (25) in order to impose a target central temperature value $\bar{T}_{c}^{t}$.

Once the temperature field $\bar{T}$ is obtained, the corresponding radiative field associated with diffuse wall emissivity values is determined. The flux $\left.\overline{q^{R}}\right|_{n c}(y)$ is computed semi-analytically in the whole channel by using integroexponential functions $[13,14]$ and by adding the contributions of each narrow band of the ck model.

\subsection{Validation of flow field}

The channel flow case of Refs. $[24,25]$ is computed from equations of Secs. 3.1-3.2. The results for the corresponding mean velocity $\bar{u}$ and temperature $\bar{T}$ are compared in Fig. 3. The temperature acts as a passive scalar and only the results with $\operatorname{Pr}=0.71$ are shown. Both the velocity and temperature profiles are predicted with a good agreement compared to the corresponding DNS results. This enables to validate a the correct implementation of the numerical strategy. 


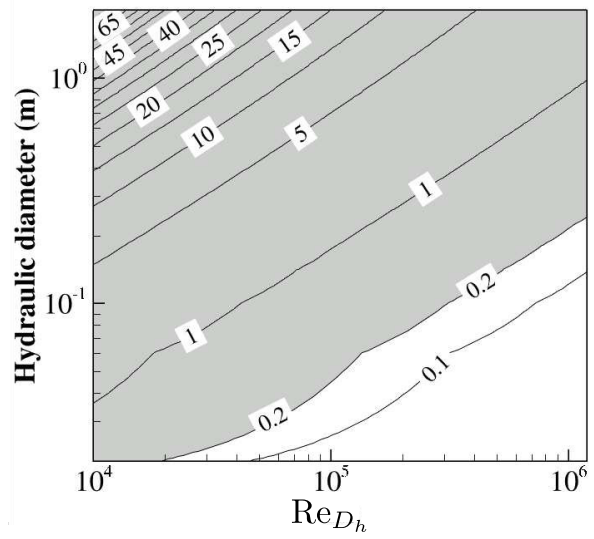

(a)

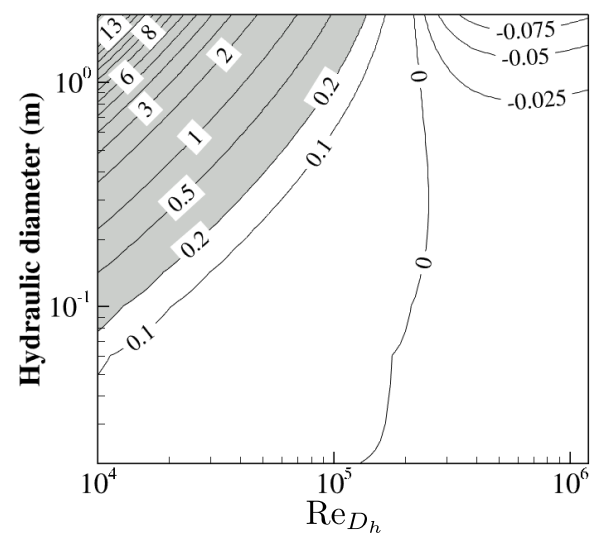

(b)

Figure 4: Contour plots of the $\widetilde{\mathcal{I}}$ (a) and $\widetilde{\mathcal{J}}$ (b) indicators as functions of the Reynolds number $\operatorname{Re}_{D_{h}}$ and the channel hydraulic diameter $D_{h}$. The pressure is set to $40 \mathrm{~atm}$ and the walls emissivity to 0.8 .

\section{Typical results of the indicators' computation}

The previous numerical approach has been used to simulate many channel flows for large ranges of hydraulic diameters of channel $D_{h}$, pressure, wall emissivity and Reynolds number (based on $D_{h}$ and the bulk velocity).

In all the cases, the wall temperatures and center line temperature are set to typical values in combustors, i.e., $800 \mathrm{~K}$ and $1800 \mathrm{~K}$ respectively. The participating medium is a non-reacting $\mathrm{CO}_{2}-\mathrm{H}_{2} \mathrm{O}-\mathrm{N}_{2}$ gas mixture, previously used in Ref. [8], of molar fractions $x_{\mathrm{CO}_{2}}=0.116, x_{\mathrm{H}_{2} \mathrm{O}}=0.155$ and $x_{\mathrm{N}_{2}}=$ 0.729. The dynamic viscosity $\mu$ is computed as a function of temperature from the CHEMKIN package [26, 27] for the chosen mixture composition. The thermal conductivity $\lambda$ is computed from the imposed Prandtl number value $\operatorname{Pr}=0.71$. The results associated with the two indicators $\widetilde{\mathcal{I}}$ and $\widetilde{\mathcal{J}}$, defined by Eqs. (3) and (16) respectively, are presented and analyzed in this section.

\subsection{Radiation effects at high pressure}

The pressure is first set to 40 atm in order to have a relatively optically thick medium and the wall emissivity to 0.8 .

The criterion $\widetilde{\mathcal{I}}$ values are plotted as a function of the channel hydraulic diameter $D_{h}$ and the Reynolds number $\operatorname{Re}_{D_{h}}$ in Fig. 4 (a). The criterion 


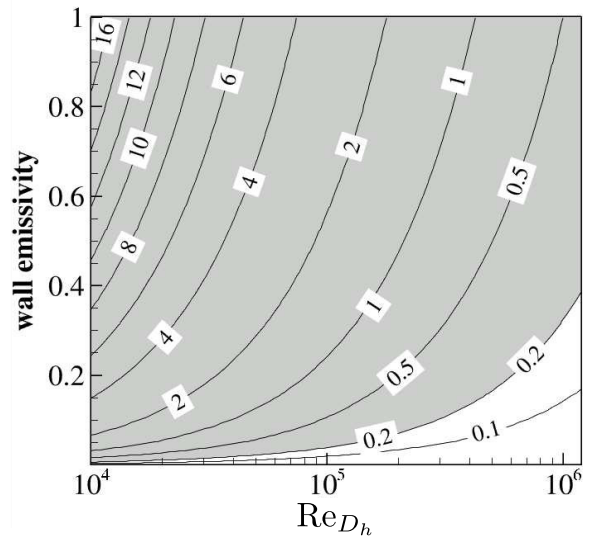

(a)

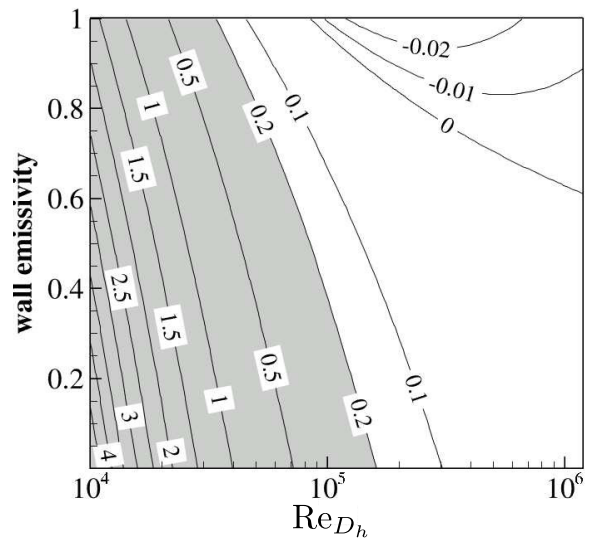

(b)

Figure 5: Contour plots of the $\widetilde{\mathcal{I}}(\mathrm{a})$ and $\widetilde{\mathcal{J}}$ (b) indicators as functions of the Reynolds number $\operatorname{Re}_{D_{h}}$ and the walls emissivity. The pressure is set to $40 \mathrm{~atm}$ and the channel hydraulic diameter $D_{h}$ to $0.4 \mathrm{~m}$.

threshold value where one must consider a radiation computation is arbitrarily chosen equal to 0.2 to simplify the discussion. It corresponds to a radiative flux equal to $20 \%$ of the conductive flux.

As expected in such a high-pressure case, the region where a radiation simulation is needed covers the main part of the domain (grey zone in the figure where $\widetilde{\mathcal{I}} \geq 0.2$ ). On the one hand, for a given Reynolds number, the importance of wall radiative flux increases with the channel hydraulic diameter of the channel, due to an increased optical thickness. On the other hand, the weight of wall radiative flux becomes less important at higher Reynolds number as the wall conductive flux increases with Reynolds number.

The criterion $\widetilde{\mathcal{J}}$ values are plotted as a function of $\operatorname{Re}_{D_{h}}$ and $D_{h}$ in the same conditions in Fig. 4 (b). A criterion threshold value of 0.2 similarly characterizes non-negligible radiation effects on the law-of-the-wall $\bar{T}^{+}\left(y^{+}\right)$: A specific coupled wall model has then to be accounted for. The grey region, such that $\widetilde{\mathcal{J}} \geq 0.2$, is smaller than the grey one associated with $\widetilde{\mathcal{I}}$ : In some region, although the wall radiative flux is important, the radiation effect is not strong enough to influence $\bar{T}^{+}$profiles near the wall.

The magnitude of the $\widetilde{\mathcal{J}}$-indicator value increases with $D_{h}$ as the optical thickness becomes larger. The dependency of $\widetilde{\mathcal{J}}$ with the Reynolds number is more complex. Two aspects have been enlightened in Ref. [8] regarding 
the influence of the Reynolds number $\operatorname{Re}_{D_{h}}$ on the wall-scaled temperature.

First, while $\operatorname{Re}_{D_{h}}$ increases, the weight of turbulent convective heat transfer increases: radiation effect is then relatively less important. Consequently, the $\bar{T}^{+}$profile gets closer to the classical law-of-the-wall, and the absolute value of $\widetilde{\mathcal{J}}$ decreases.

On the other hand, the balance between gas-gas and gas-wall radiative contributions modifies the impact of radiation on the system. As the temperature gradient in the vicinity of the channel walls increases with Reynolds number, the difference between a fluid point and the wall temperatures increases with $\mathrm{Re}_{D_{h}}$; On the contrary, the difference between the same point temperature and the gas bulk temperature decreases. Consequently, the weight of gas-gas radiation is reduced while the gas-wall radiation effect is enhanced. Moreover, as discussed in Ref. [8], gas-gas radiation decreases the $\bar{T}^{+}$value and yields a positive $\widetilde{\mathcal{J}}$ value whereas gas-wall radiation has an opposite effect. Therefore, as Reynolds number increases, the value of the $\widetilde{\mathcal{J}}$ criterion is expected to decrease and to even become negative when gas-wall radiation dominates over gas-gas radiation. The latter effect $(\widetilde{\mathcal{J}}<0)$, that has been anticipated from DNS studies but could not be observed due to the computational limitations associated with high Reynolds numbers, is clearly shown in the top-right corner of Fig. 4 (b). For smaller Reynolds number values, gas-gas radiation is dominant and a positive value of $\widetilde{\mathcal{J}}$ is obtained. Both of the two aforementioned aspects tend to decrease the criterion value when Reynolds number increases.

The influences of the Reynolds number $\operatorname{Re}_{D_{h}}$ and wall emissivity on the two indicators $\widetilde{\mathcal{I}}$ and $\widetilde{\mathcal{J}}$, for a pressure of $40 \mathrm{~atm}$ and a channel hydraulic diameter $D_{h}$ of $0.4 \mathrm{~m}$, are shown in Fig. 5. As expected, the radiative flux, and hence the $\widetilde{\mathcal{I}}$ indicator, increases with the wall emissivity. On the other hand, the effects of the wall emissivity on the $\bar{T}^{+}$profile is again understood through its impact on the balance between gas-gas and gas-wall radiation: as the wall emissivity increases, gas-wall radiation is enhanced and the weight of gas-gas radiation decreases. Consequently, the value of the indicator $\widetilde{\mathcal{J}}$ decreases with the wall emissivity from a high positive value to a possible negative value, reached for a high Reynolds number.

The maximum effects of radiation on each indicator are not located in the same region of Figs. 4 and 5. The maximum value of $\widetilde{\mathcal{I}}$ appears in the topleft corner (small $\operatorname{Re}_{D_{h}}$, high wall emissivity) while the maximum value of $\widetilde{\mathcal{J}}$ 


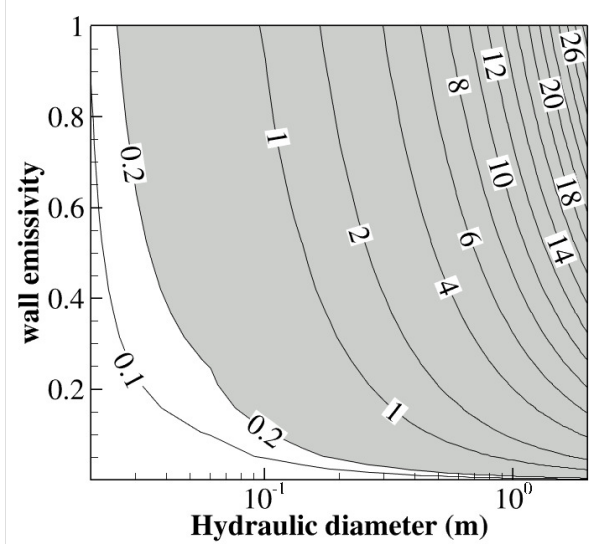

(a)

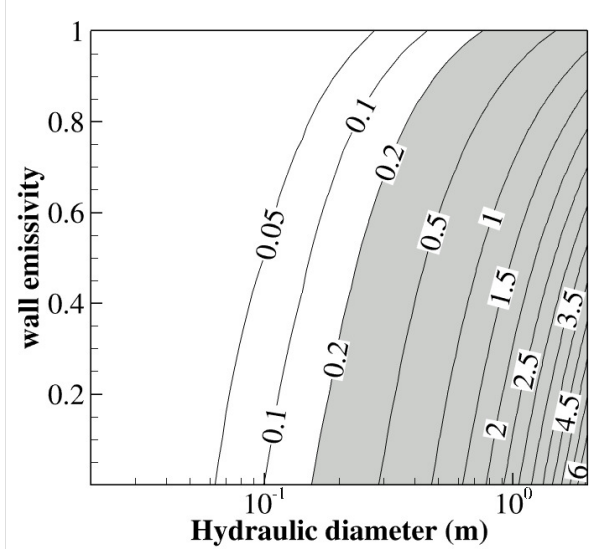

(b)

Figure 6: Contour plots of the $\widetilde{\mathcal{I}}$ (a) and $\widetilde{\mathcal{J}}$ (b) indicators as function of the walls emissivity and the channel hydraulic diameter $D_{h}$. The pressure is set to $40 \mathrm{~atm}$ and $\left.\operatorname{Re}_{D_{h}}=48,000\right)$.

is in the bottom-left corner (small $\mathrm{Re}_{D_{h}}$, small wall emissivity). Therefore, maximizing the wall radiative flux does not necessarily imply a maximum effect on the mean temperature profile. Indeed, the modification of the scaled temperature profile $\bar{T}^{+}$defined in Eq. 6 is mainly due to the wall conductive flux which, as previously shown [8], decreases with gas-wall radiation and increases with gas-gas radiation. Hence, the wall emissivity has opposite effects on the wall conductive and radiative fluxes: wall conductive flux decreases with wall emissivity whereas the wall radiative flux, on the contrary, increases. When both fluxes have the same order of magnitude, a careful consideration of radiative effects is consequently needed for determining the impact of wall emissivity on the total heat flux.

The influences of the channel hydraulic diameter $D_{h}$ and the wall emissivity on the two indicators $\widetilde{\mathcal{I}}$ and $\widetilde{\mathcal{J}}$, for a pressure of $40 \mathrm{~atm}$ and a Reynolds number $\operatorname{Re}_{D_{h}}$ value of 48,000, are shown in Fig. 6. As previously discussed, both indicator values increase with $D_{h}$, while they show opposite trends with the wall emissivity. Therefore, critical values appear in the top-right corner for $\widetilde{\mathcal{I}}$ and on the bottom-right corner for $\widetilde{\mathcal{J}}$. 


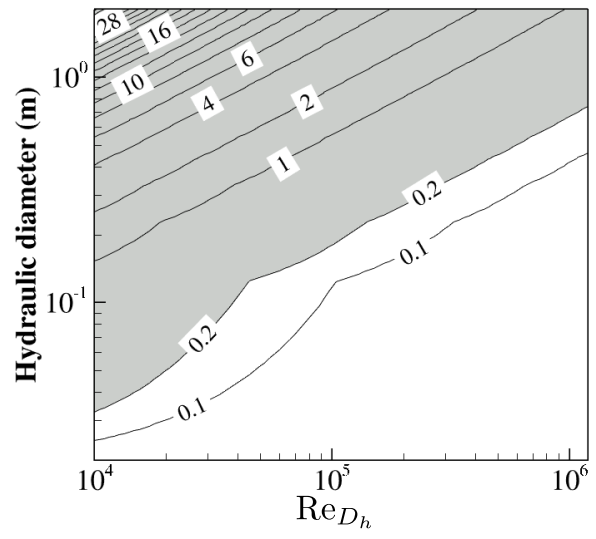

(a)

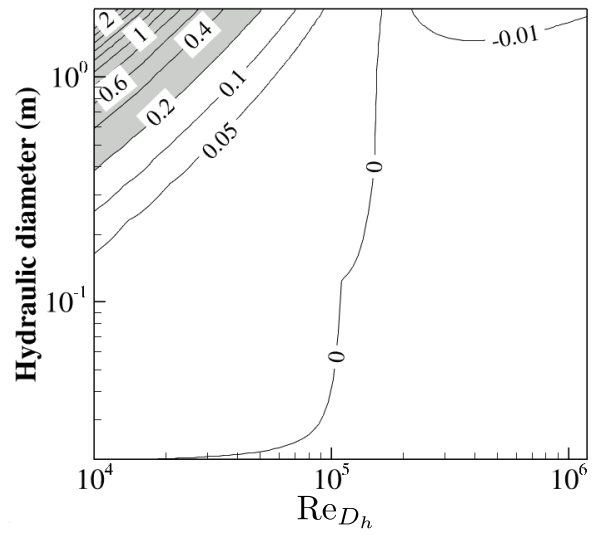

(b)

Figure 7: Contour plots of the $\widetilde{\mathcal{I}}$ (a) and $\widetilde{\mathcal{J}}$ (b) indicators as functions of the practical Reynolds number $\operatorname{Re}_{D_{h}}$ and the channel hydraulic diameter $D_{h}$. The pressure is set to 1 atm and the walls emissivity to 0.8 .

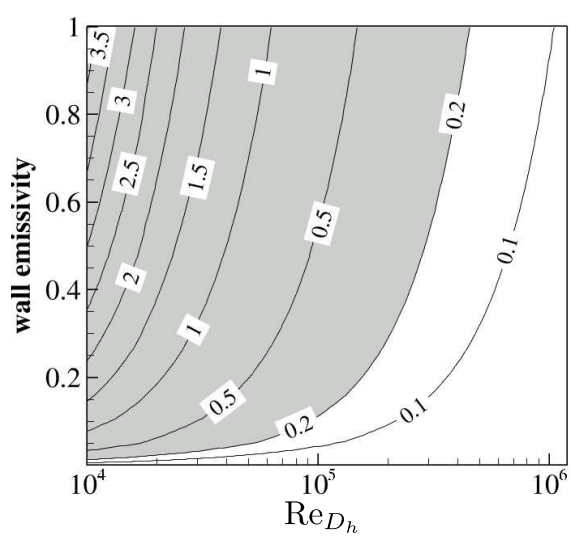

(a)

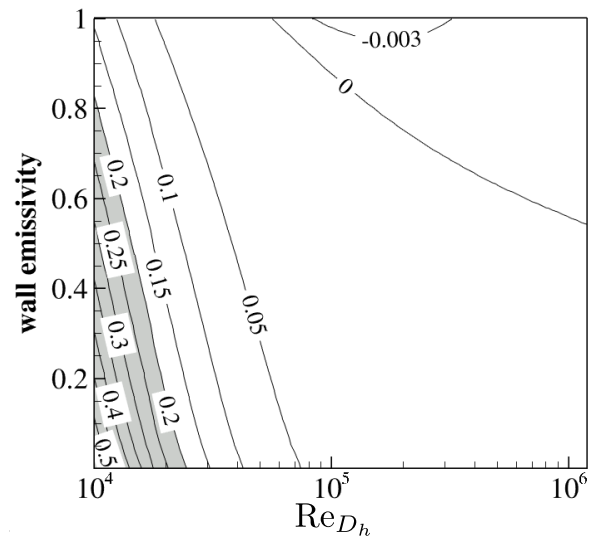

(b)

Figure 8: Contour plots of the $\widetilde{\mathcal{I}}$ (a) and $\widetilde{\mathcal{J}}$ (b) indicators as functions of the Reynolds number $\operatorname{Re}_{D_{h}}$ and the walls emissivity. The pressure is set to $1 \mathrm{~atm}$ and the channel hydraulic diameter $D_{h}$ to $0.4 \mathrm{~m}$. 


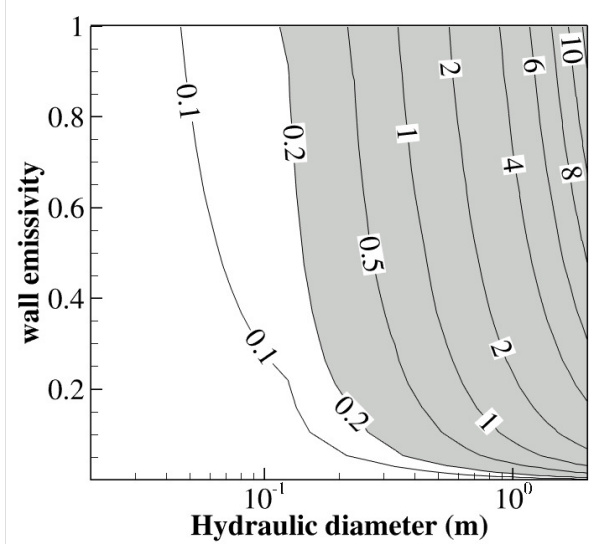

(a)

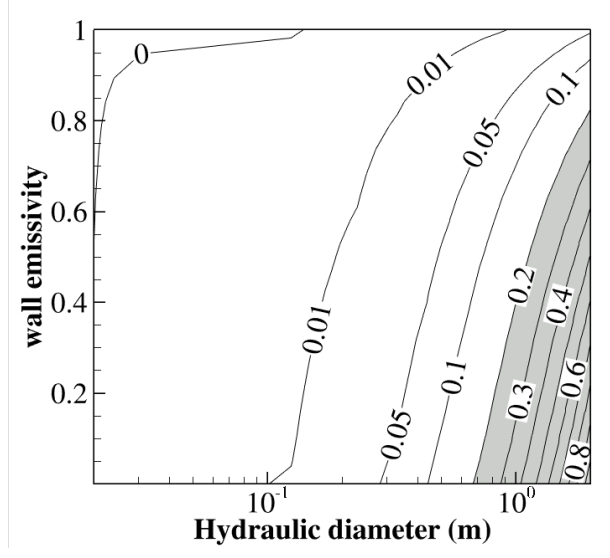

(b)

Figure 9: Contour plots of the $\widetilde{\mathcal{I}}$ (a) and $\widetilde{\mathcal{J}}$ (b) indicators as functions of the walls emissivity and the channel hydraulic diameter $D_{h}$. The pressure is set to 1 atm and $\operatorname{Re}_{D_{h}}=48,000$.

\subsection{Radiation effects at atmospheric pressure}

The indicators $\tilde{\mathcal{I}}$ and $\tilde{\mathcal{J}}$ are plotted in Figs. 7, 8 and 9 for different flow conditions under the same pressure of $1 \mathrm{~atm}$. Observed trends are similar to those corresponding to $40 \mathrm{~atm}$ although the indicator value magnitudes and the sizes of the critical regions decrease as the optical thickness at 1 atm is much smaller than at $40 \mathrm{~atm}$, for a given data set. Unsurprisingly, smaller radiation effects are then observed at $1 \mathrm{~atm}$.

\subsection{Influence of pressure on radiation effects}

Since the gas absorption coefficients increases with pressure, stronger radiation effects are achieved for the largest pressure values, as shown in Figs. 10, 11 and 12 where both indicators are plotted.

\section{Conclusions}

Two practical indicators have been proposed to characterize radiation effects in turbulent gaseous boundary layers. The first one estimates the weight of wall radiative flux compared to the wall conductive flux. It allows to define (within a given accuracy) the conditions for which the wall radiative flux cannot be neglected. The more advanced second indicator characterizes the magnitude of coupling effects between conduction, turbulent convection 


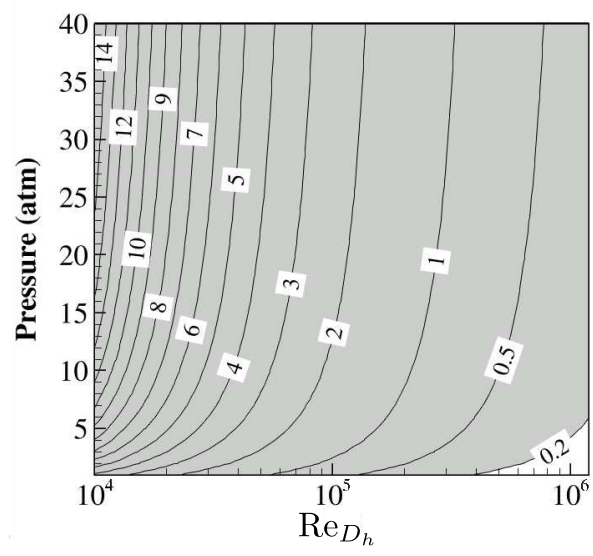

(a)

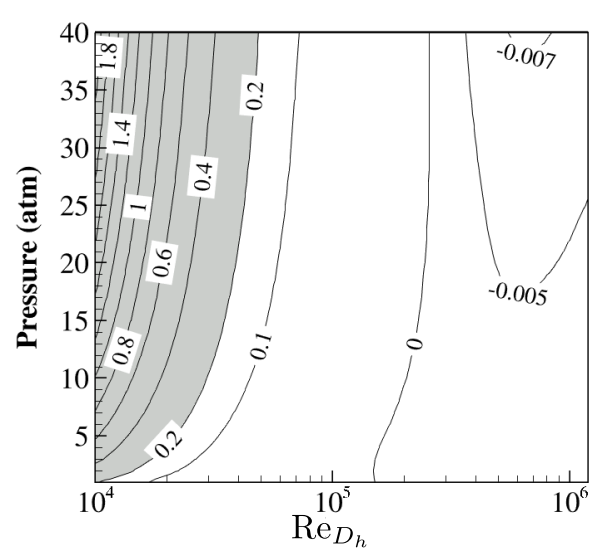

(b)

Figure 10: Contour plots of the $\widetilde{\mathcal{I}}$ (a) and $\widetilde{\mathcal{J}}$ (b) indicators as functions of the Reynolds number $\operatorname{Re}_{D_{h}}$ and pressure. The channel hydraulic diameter $D_{h}$ is set to $0.4 \mathrm{~m}$ and the walls emissivity to 0.8 .



(a)

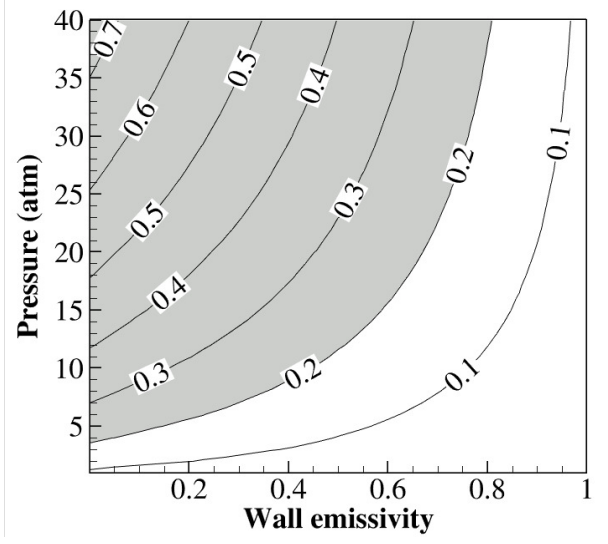

(b)

Figure 11: Contour plots of the $\widetilde{\mathcal{I}}(\mathrm{a})$ and $\widetilde{\mathcal{J}}(\mathrm{b})$ indicators as functions of the walls emissivity and pressure. The channel hydraulic diameter is set to $0.4 \mathrm{~m}$ and $\operatorname{Re}_{D_{h}}=$ $48,000$. 


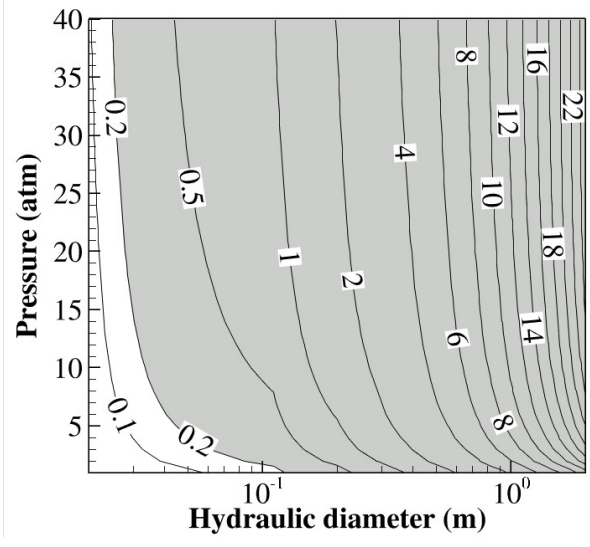

(a)

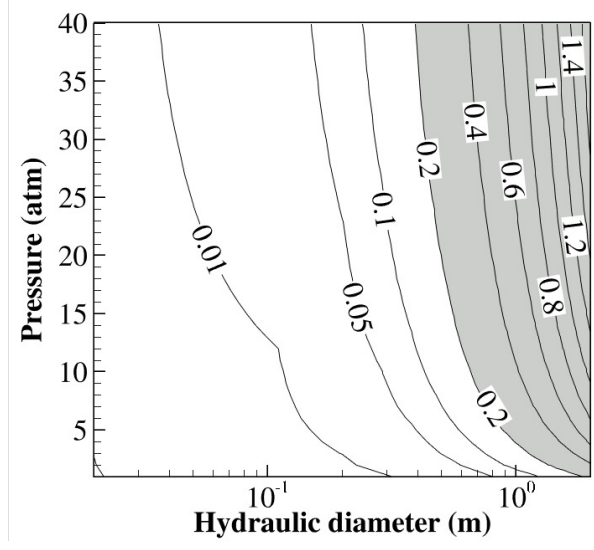

(b)

Figure 12: Contour plots of the $\widetilde{\mathcal{I}}$ (a) and $\widetilde{\mathcal{J}}$ (b) indicators as functions of the channel hydraulic diameter $D_{h}$ and pressure. The walls emissivity is 0.8 and $\operatorname{Re}_{D_{h}}=48,000$.

and radiation on the temperature law-of-the-wall within the gaseous boundary layer. In such conditions, the near-wall mean temperature profile and the wall conductive flux are modified. It is then necessary to implement a specific turbulent wall model, as done in Ref. [12], to account for radiation effects on the temperature law-of-the-wall in the near wall region.

Both practical indicators are based on mean flow velocity and temperature fields issued from a fast simulation performed without radiation, i.e. from the uncoupled solution of a set of 1D RANS equations. The fields of radiative flux and power are then computed from the obtained mean profiles. Consequently, turbulence-radiation interactions are not taken into account in the simplified formulation of the indicators. Nonetheless, these indicators have been validated by comparison with coupled DNS/Monte-Carlo results which account for detailed reciprocal turbulence-radiation couplings. The simplified indicators allow then to determine when a complete DNS-Monte Carlo calculation is not necessary or has to be carried out for accounting for all coupling effects, including reciprocal turbulence-radiation interactions, as done in a previous publication [8].

All the results have been determined for a typical mixture of combustion products within a plane channel, of which the spectral radiative properties are accurately described by a ck method. Computed conditions are characterized by four parameters: pressure, channel hydraulic diameter, associated 
Reynolds number and assumed grey wall emissivity. The large number of performed computations allows a complete mapping of both indicators under large ranges of parameter values, including high pressure and atmospheric pressure.

The resulting observations have confirmed and generalized the conclusions obtained from previously analyzed DNS data. When the medium optical thickness increases (when the pressure or system size increases), radiation effect is enhanced and higher indicator values are obtained. When wall emissivity is modified, the trends associated to the two criteria are opposite since the wall radiative flux is mainly due to gas-wall radiation while a modification of the wall-scaled temperature is generally dominated by gas-gas radiation. Both practical indicators decrease with Reynolds number and a small negative value of the indicator for wall-scaled temperature is observed when gas-wall radiation overcomes gas-gas radiation in flows characterized by high Reynolds number.

Future works should be carried out to also derive an a priori estimation of the reciprocal effects of turbulence-radiation fluctuations in order to quantify this additional coupling effect in turbulent flows. The investigated conditions typically in the range $800-2500 \mathrm{~K}$ are typical of combustion applications. At higher temperatures, if the gas remains in Local Thermal Equiliubrium (LTE), the framework of the derived indicators still holds. In the case of a gaseous medium in non-LTE conditions, a dedicated study has to be carried out for analyzing the effects of radiation in such turbulent boundary layers.

\section{Acknowledgements}

This work was granted access to the HPC resources of IDRIS under the allocations 2013-2b0164 made by GENCI (Grand Equipement National de Calcul Intensif).

\section{References}

[1] A. Soufiani, P. Mignon, J. Taine, Radiation effects on turbulent heat transfer in channel flows of infrared active gases, Proceedings of the AIAA/ ASME thermophysics and heat transfer conference, 1990.

[2] J. Amaya, O. Cabrit, D. Poitou, B. Cuenot, M. El Hafi, Unsteady coupling of Navier-Stokes and radiative heat transfer solvers applied to 
an anisothermal multicomponent turbulent channel flow, Journal of Quantitative Spectroscopy and Radiative Transfer 111 (2010) 295-301.

[3] D. Poitou, J. Amaya, C. Bushan Singh, D. Joseph, M. El Hafi, B. Cuenot, Validity limits for the global model fs-snbck for combustion applications, Proceedings of Eurotherm83 - Computational Thermal Radiation in Participating Media III, 2009.

[4] A. Gupta, M. F. Modest, D. C. Haworth, Large-Eddy Simulation of Turbulence-Radiation Interactions in a Turbulent Planar Channel Flow, Journal of Heat Transfer-Transactions of the ASME 131 (2009) 061704.

[5] S. Ghosh, R. Friedrich, M. Pfitzner, C. Stemmer, B. Cuenot, M. El Hafi, Effects of radiative heat transfer on the structure of turbulent supersonic channel flow, Journal of Fluid Mechanics 677 (2011) 417-444.

[6] F. Liu, G. J. Smallwood, Ö. L. Gülder, Application of the statistical narrow-band correlated-k method to low-resolution spectral intensity and radiative heat transfer calculations - effects of the quadrature scheme, International Journal of Heat and Mass Transfer 43 (2000) 3119-3135.

[7] A. Sakurai, K. Matsubara, K. Takakuwa, R. Kanbayashi, Radiation effects on mixed turbulent natural and forced convection in a horizontal channel using direct numerical simulation, International Journal of Heat and Mass Transfer 55 (2012) 2539-2548.

[8] Y. F. Zhang, R. Vicquelin, O. Gicquel, J. Taine, Physical study of radiation effects on the boundary layer structure in a turbulent channel flow, International Journal of Heat and Mass Transfer 61 (2013) 654666.

[9] Y. F. Zhang, O. Gicquel, J. Taine, Optimized emission-based reciprocity monte carlo method to speed up computation in complex systems, International Journal of Heat and Mass Transfer 55 (2012) 8172-8177.

[10] R. Goody, R. West, L. Chen, D. Crisp, The correlated-k method for radiation calculations in nonhomogeneous atmospheres, Journal of Quantitative Spectroscopy and Radiative Transfer 42 (1989) 539 - 550. 
[11] A. Soufiani, J. Taine, High temperature gas radiative property parameters of statistical narrow-band model for $\mathrm{H}_{2} \mathrm{O}, \mathrm{CO}_{2}$ and $\mathrm{CO}$, and correlated-K model for $\mathrm{H}_{2} \mathrm{O}$ and $\mathrm{CO}_{2}$, International Journal of Heat and Mass Transfer 40 (1997) $987-991$.

[12] R. Vicquelin, Y. F. Zhang, O. Gicquel, J. Taine, Effects of radiation in turbulent channel flow: analysis of coupled direct numerical simulations, Journal of Fluid Mechanics 753 (2014) 360-401.

[13] Y. F. Zhang, R. Vicquelin, O. Gicquel, J. Taine, A wall model for les accounting for radiation effects, International Journal of Heat and Mass Transfer 67 (2013) 712-723.

[14] M. F. Modest, Radiative Heat Transfer, Academic Press, 2003.

[15] P. J. Coelho, Numerical simulation of the interaction between turbulence and radiation in reactive flows, Progress in Energy and Combustion Science 33 (2007) 311-383.

[16] H. Schlichting, K. Gersten, Boundary layer theory, Springer, 8th edition, 2000 .

[17] M. C. W.M. Kays, B. Weigand, Convective Heat and Mass Transfer, McGraw-Hill, 2004.

[18] G. Coleman, J. Kim, R. Moser, A numerical study of turbulent supersonic isothermal-wall channel flow, J. Fluid Mech. 305 (1995) 159-183.

[19] A. Patel, J. W. R. Peeters, B. J. Boersma, R. Pecnik, Semi-local scaling and turbulence modulation in variable property turbulent channel flows, Physics of Fluids 27 (2015) 095101.

[20] P. Huang, G. Coleman, P. Bradshaw, Compressible turbulent channel flows: DNS results and modelling, Journal of Fluid Mechanics 305 (1995) $185-218$.

[21] K. Abe, T. Kondoh, Y. Nagano, A new turbulence model for predicting fluid flow and heat transfer in separating and reattaching flows - ii. thermal field calculations, International Journal of Heat and Mass Transfer 38 (1995) 1467-1481. 
[22] S. V. Patankar, Numerical heat transfer and fluid flow, Hemishpere Pub. Corp., New York, 1980.

[23] W. M. Kays, Turbulent Prandtl number. Where are we?, Journal of Heat Transfer-Transactions of the ASME 116 (1994) 284-295.

[24] J. Kim, P. Moin, R. Moser, Turbulence statistics in fully developed channel flow at low Reynolds number, Journal of Fluid Mechanics 177 (1987) 133-166.

[25] J. Kim, P. Moin, Transport of passive scalars in a turbulent channel flow, Turbulent Shear Flows 6th, 1987.

[26] R. J. Kee, G. Dixon-lewis, J. Warnatz, M. E. Coltrin, J. A. Miller, A Fortran Computer Code Package For The Evaluation Of Gas-Phase, Multicomponent Transport Properties, Technical Report SAND89-8009, Sandia National Laboratories, 1986.

[27] R. J. Kee, F. M. Rupley, J. A. Miller, CHEMKIN-II: A Fortran Chemical Kinetics Package for the Analysis of Gas-Phase Chemical Kinetics, Technical Report SAND89-8009, Sandia National Laboratories, 1989. 